\title{
Custo das doenças atribuíveis a fatores ambientais na cidade de Manaus, Amazonas, Brasil
}

\author{
Cost of illness attributable to environmental factors \\ in the city of Manaus, state of Amazonas, Brazil
}

\author{
Marcilio Sandro de Medeiros ${ }^{1}$ \\ Daniel Souza Sacramento ${ }^{1}$ \\ José Camilo Hurtado-Guerrero ${ }^{2}$ \\ Ramon Arigoni Ortiz ${ }^{3}$ \\ André Luiz Dutra Fenner ${ }^{4}$
}

${ }^{1}$ Instituto Leônidas e Maria Deane, Fundação Oswaldo Cruz. R. Teresina 476, Adrianópolis. 69.057-070 Manaus AM Brasil. muribeka@amazonia.fiocruz.br

${ }^{2}$ Escola Superior de Saúde, Universidade do Estado do Amazonas.

${ }^{3}$ Estudos Econômicos em Sustentabilidade. ${ }^{4}$ Centro Colaborador da OPAS/OMS em Saúde Pública e Ambiental, Fundação Oswaldo Cruz.
Abstract The study estimated the cost of illness attributable to environmental factors in the city of Manaus between the years 1998 to 2009. The causes of hospitalization were grouped based on studies of the Global Burden of Disease and Comparative Risk Assessment of the World Health Organization. The value was estimated by the sum of (i) hospital spending on treatment of diseases directly attributable to environmental factors, and (ii) the costs of lost workdays resulting from the stay in hospital estimated on the basis of the average earnings of Manaus workers. The data were further calibrated taking into account the coverage of the population with private health insurance. The cost of illness, considering the values corrected by the General Market Price Index for the year 2009 was estimated at $R \$ 286,852,666.97$, of which cardiovascular disease, respiratory infections of the lower airways and diarrheal diseases are responsible for $78.6 \%$ of these values. Of the fractions attributable to environmental factors, cardiovascular diseases account for 16\% (CI: 7$23 \%)$, respiratory infections and respiratory infections of the lower airways for 41\% (CI: 32-47\%), and diarrhea for $94 \%$ (CI: 84-98\%) of the global burden of disease.

Key words Morbidity, Costs of health care, Health economics
Resumo O presente estudo estimou o custo das doenças atribuíveis a fatores ambientais na cidade de Manaus, entre os anos de 1998 a 2009. As causas de internação hospitalar foram agrupadas com base nos estudos de Carga Global de Doença e da Análise Comparativa de Risco da Organização Mundial da Saúde. O valor foi estimado por meio da soma dos (i) gastos hospitalares com tratamento das doenças atribuiveis a fatores ambientais com os (ii) valores dos dias de trabalho perdidos resultante da permanência em leito hospitalar, estimados a partir da remuneração média dos trabalhadores de Manaus. Os dados ainda foram calibrados levando-se em consideração a cobertura da população com plano de saúde privado. O custo das doenças, considerando os valores corrigidos pelo Índice Geral de Preços do Mercado para o ano de 2009, foi estimado em $R \$ 286.852 .666,97$, dos quais as doenças cardiovasculares, as infecções respiratórias das vias aéreas inferiores e as doenças diarreicas são responsáveis por 78,6\%. Das frações atribuiveis a fatores ambientais, as doenças cardiovasculares respondem por 16\% (IC: 7-23\%), as infecções respiratórias das vias aéreas inferiores por $41 \%$ (IC: $32-47 \%$ ), e as diarreias por $94 \%$ (IC: 84-98\%) da carga global das doenças.

Palavras-chave Morbidade, Custos de cuidados de saúde, Economia da saúde 


\section{Introdução}

Na Amazônia Legal os diferentes processos de ocupação e desenvolvimento socioeconômico têm possibilitado a melhoria de alguns indicadores tradicionais de saúde e bem-estar, como, por exemplo, a redução da mortalidade infantil e o aumento da expectativa de vida. Por outro lado, o desenvolvimento também implica diversos custos ao ser humano, dentre eles os associados à degradação ambiental e a hábitos de vida não saudáveis, com forte repercussão na saúde humana ${ }^{1-4}$.

Prüss-Üstün e Corvalán ${ }^{5}$, ao analisarem mais detalhadamente os fatores de risco ambientais que agem sobre o processo saúde-doença pelo mundo, constataram que 85 de 102 enfermidades investigadas eram atribuíveis especificamente às mudanças ambientais. No mundo, $24 \%$ dos anos de vida perdidos por incapacidade e $23 \%$ por morte prematura foram atribuídas a fatores ambientais.

As doenças fortemente atribuíveis ao estilo de vida são as que mais crescem pelo mundo. Em 2005, Portugal contabilizou 191 milhões de euros os gastos diretos com saúde atribuíveis ao consumo de álcool, sendo $50 \%$ do total (95.141.391 milhões de euros) gastos com internação hospitalar ${ }^{6}$. Em geral, a balança comercial relativa aos ganhos econômicos do setor produtor de bebidas destiladas e o ônus dos custos sociais das despesas com assistência médico-hospitalar é deficitária. Os Estados Unidos em 1995 arrecadaram em impostos com a venda de bebidas alcoólicas US\$ 20 bilhões e arcaram com US\$ 40 bilhões em gastos com saúde associadas ao consumo de álcool ${ }^{7}$.

O sedentarismo é outro fator de risco relacionado às diversas enfermidades crônicas-degenerativas, entre elas a cardiopatia isquêmica, o câncer de mama, o câncer de colón, o câncer de reto e a diabetes mellitus. Na América do Norte, estima-se que a frequência dessas patologias poderia ser reduzida em $31 \%$ mediante intervenções ambientais planejadas, tais como construção de áreas de lazer e esporte, melhoria do sistema de mobilidade das cidades (ciclovias) e espaços de convivência no trabalho ${ }^{5}$.

Em 1997, a Companhia de Tecnologia de Saneamento Ambiental de São Paulo (Cetesb) em parceria com o Instituto de Pesquisa Econômica Aplicada (Ipea) estimou por meio de um indicador composto o impacto associado à saúde humana causado pela poluição atmosférica na Região Metropolitana de São Paulo (RMSP). O indicador de morbidade foi obtido a partir da soma dos gastos hospitalares totais por doenças respiratórias e cardiovasculares com o valor dos dias de trabalho perdidos devido à doença, calculado com base nos salários médios na região. O valor convertido em moeda nacional e corrigido pelo Índice Geral de Preços do Mercado para o ano de 2009 foi estimado em $\mathrm{R} \$ 237.414 .869,39$, dos quais as doenças cardiovasculares representaram $51,9 \%$ dos custos para sociedade ${ }^{3}$.

$\mathrm{Na}$ literatura científica internacional existe uma relativa quantidade de referências bibliográficas relacionadas à análise dos custos econômicos e sociais dos problemas de saúde mais prevalentes nas populações. Carvalho e Cerqueira ${ }^{8}$ contabilizaram mais de 60 artigos sobre valores monetários do risco de mortalidade (mortality risk premium) e aproximadamente 40 estudos sobre valores monetários do risco de danos físicos (injury risk premium). As expressões mais recorrentes aplicadas a esses estudos são valor estatístico da vida, perda de produção devido à morte, valores de referência para a vida humana e custos de cuidados de saúde, todos desenvolvidos sobre a temática da economia da saúde. Em geral, os estudos se dedicam a estimar as perdas de produtividade causadas pelos anos de vida perdidos por morte prematura (APVP) e os anos de vida perdidos por incapacidade (AVPI).

No Brasil, só recentemente foram publicados estudos no intuito de estimular o debate acadêmico e chamar a atenção para a necessidade de se estabelecer e difundir metodologias de aferição e de análise dos custos econômicos e sociais oriundos do saneamento inadequado, poluição do ar, homicídios e acidentes de trânsito. Portanto, não há na literatura especializada pesquisas que avaliem os custos das doenças relacionados a fatores ambientais nas cidades brasileiras. Nesse sentido, objetivou-se estimar o custo das doenças fortemente atribuíveis a fatores ambientais na cidade de Manaus, capital do estado do Amazonas. Para isso foi necessário calcular os gastos hospitalares atribuíveis a fatores ambientais e quantificar os dias de trabalho perdidos não remunerados resultantes das enfermidades.

\section{Materiais e métodos}

\section{As doenças fortemente atribuíveis a fatores ambientais}

As doenças fortemente atribuíveis a fatores ambientais definidas neste estudo foram propostas por Prüss-Üstün e Corvalán e estão destaca- 
das no Quadro 1. No entanto, três questões importantes devem ser relativizadas para a compreensão das doenças atribuíveis a fatores ambientais $^{5,9}$.

A primeira, que está diretamente ligada ao campo epistemológico, atribui à disciplina saúde ambiental o estudo das enfermidades oriundas do meio ambiente. Para tanto, é necessário desfazer a ideia limitada de que as causas ambientais das doenças são todas aquelas de etiologia não genética, como, também, eleger somente as doenças do meio ambiente de contacto com alguns órgãos do corpo humano via ingestão, inalação ou penetração. Tal pressuposto origina-se da clássica dicotomia entre "natureza" e "natural". Nessa abordagem, entre os fatores ambientais se incluem todos aqueles que afetam direta ou indireta- mente o organismo humano após sua concepção, inclusive aqueles originados no ambiente social. Nesse sentido, pode-se afirmar que fatores genéticos são, na verdade, também ambientais.

A segunda, mais importante na atualidade, em termos de promoção da saúde ou saúde e qualidade de vida, afeta distintas etnias e nacionalidades pelo mundo. Esta diz respeito à compreensão das mudanças do comportamento humano ou do estilo de vida dos indivíduos responsáveis pela inclusão de uma gama de novos fatores de risco na humanidade (alcoolismo, tabagismo, homicídio, acidente por tráfico rodoviário, estresse, guerras...). De certo modo, é possível que esses fatores de risco originados no ambiente social tendam a ser secundarizados em virtude do clássico entendimento de que fatores ambientais

Quadro 1. Doenças e agravos fortemente atribuíveis a fatores ambientais. Estimativa da fração atribuível.

\begin{tabular}{|c|c|c|c|c|c|c|c|c|c|c|c|c|}
\hline Fatores de risco & 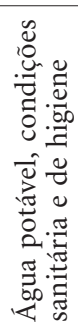 & 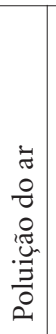 & 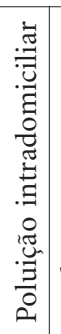 & 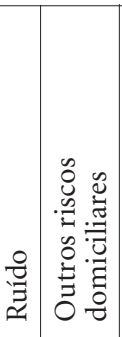 & 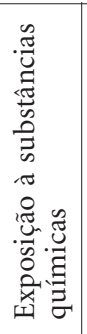 & 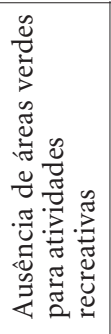 & 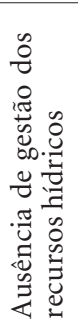 & 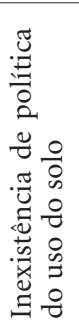 & 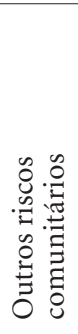 & 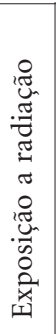 & 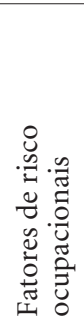 & 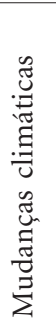 \\
\hline \multicolumn{13}{|l|}{ Tuberculose } \\
\hline Doença Diarreica & & & & & & $\bigcirc$ & & & & & & $\bigcirc$ \\
\hline Hepatite B & & & & & & & & & & & $\bigcirc$ & \\
\hline Hepatite C & & & & & & & & & & & $\bigcirc$ & \\
\hline Malária & & & & & & & & & O & & $\bigcirc$ & $\bigcirc$ \\
\hline Dengue & & & & & & & & & & & & $\bigcirc$ \\
\hline $\begin{array}{l}\text { Infecções respiratórias das vias aéreas } \\
\text { inferiores }\end{array}$ & & & $\bigcirc$ & $\bigcirc$ & & & & & & & & \\
\hline Má nutrição proteico-energética & & & & & & & & & $\bigcirc$ & & & $\bigcirc$ \\
\hline \multicolumn{13}{|l|}{ Câncer de traquéia, brônquios e pulmão } \\
\hline \multicolumn{13}{|l|}{ Consumo de drogas e álcool } \\
\hline \multicolumn{13}{|l|}{ Doença de Parkinson } \\
\hline Doenças cardiovasculares & $\bigcirc$ & 0 & $\bigcirc 6$ & $\bigcirc$ & $\bigcirc$ & $\bigcirc$ & & $\bigcirc$ & & & O & $\bigcirc$ \\
\hline Doença pulmonar obstrutiva crônica & & O & $\bigcirc$ & & & & & & & & 0 & \\
\hline Asma & & O & $\bigcirc$ & $\bigcirc$ & & & & & & & O & \\
\hline \multicolumn{13}{|l|}{ Acidente de trânsito } \\
\hline Quedas & & & & 0 & & $\bigcirc$ & & $\bigcirc$ & O & & 0 & \\
\hline Violência & & & & O & $\bigcirc$ & & & O & & & & \\
\hline \multicolumn{13}{|l|}{ Legenda: } \\
\hline
\end{tabular}

Fonte: Prüss-Üstün e Corvalán ${ }^{5}$ 
são apenas os oriundos do meio ambiente, concepção que não adotamos neste estudo.

A terceira e última questão emana das ciências matemáticas e parte da compreensão do que vem a ser "fração de risco atribuível a fatores ambientais". Resumidamente, esta é a porcentagem de uma particular categoria de doença que poderia ser evitada caso fossem eliminados os fatores de risco atribuíveis a ela. Em outras palavras, as internações atribuíveis a fatores ambientais referem-se àqueles fatores ambientais enunciados como causadores das hospitalizações. Diversamente, a expressão 'internações associadas' abarca todas as hospitalizações por doenças associadas, em algum grau, aos fatores ambientais de interesse. Logo, esta última categoria de internações constitui um número bem maior do que o número de internações atribuíveis a fatores ambientais ${ }^{9,10}$.

Em suma, o quadro no anexo originou-se de um projeto desenvolvido pela Organização Mundial da Saúde (OMS) denominada de Análise Comparativa de Risco (ACR). A metodologia baseou-se em uma profunda revisão bibliográfica de mais de duzentos títulos e da participação de mais de cem especialistas em um painel internacional que forneceram as melhores estimativas das frações atribuíveis ao ambiente das doenças às quais se dedicam ${ }^{5}$.

As doenças e os agravos ora descritas são de conhecimento técnico-científico, em uma perspectiva de causalidade, o que permitiria, por meio de medidas sanitárias, por exemplo, a redução e o controle das mesmas. Nesse sentido, como vários tipos de doenças são impactadas por fatores ambientais, a análise forja uma nova base de compreensão das interações entre o ambiente e a saúde das populações ${ }^{5,11}$.

\section{Morbidade hospitalar segundo classificação da Carga Global de Doença}

As causas de internação hospitalar foram agrupadas segundo classificação alternativa da décima Classificação Internacional das Doenças (CID10) proposta pela OMS no estudo de Carga Global de Doença (Global Burden of DiseaseGBD $)^{12}$ e posteriormente destacadas por PrüssÜstün e Corvalán ${ }^{5}$. A nova classificação as distribuiu em três grandes grupos - G1: infecciosas e parasitárias, condições perinatais e deficiências nutricionais; G2: não transmissíveis; G3: causas externas \% as doenças e agravos fortemente atribuíveis a fatores ambientais ou ao meio ambiente (FAMA).
O delineamento do estudo é do tipo transversal e descritivo, sendo as informações obtidas a partir da análise de dados secundários coletados nas guias de autorização de internação hospitalar disponíveis no Sistema de Informação Hospitalar no site do Departamento de Informática do Ministério da Saúde (SIH/Datasus) no período de 1998 a 2009.

\section{Plano de Análise do Custo da Doença}

O plano de análise para obtenção do custo da doença utilizou-se da abordagem simplificada proposta por Motta et $\mathrm{al}^{3}$. O custo monetário, ou custo das doenças (CD), foi estimado por meio da soma de dois componentes: (i) gastos hospitalares com tratamento das doenças fortemente atribuíveis a fatores ambientais; (ii) valores dos dias de trabalho perdidos resultantes da permanência em leito hospitalar.

O valor dos dias de trabalho perdidos, ou não remunerados, em virtude da internação hospitalar foi calculado com base nos dados da remuneração média dos trabalhadores de Manaus disponível no site da Relação Anual de Informações Sociais (RAIS) do Ministério do Trabalho e Emprego (MTE). Nesse cálculo, considerou-se, ainda, para cada ano analisado, a cobertura da população com plano de saúde privado, acrescido da população de estudo entre os anos 1998 a 2009 ( $\mathrm{N}=200.393$ internações por FAMA). O dado foi obtido no site da Agência Nacional de Saúde Suplementar (ANS). Em virtude da ausência de dados sobre a cobertura de plano de saúde privado para os dois primeiros anos para a cidade de Manaus, replicou-se a cobertura do ano subsequente mais próximo. Sumariamente, a estimativa é representada pela expressão matemática logo abaixo.

Fórmula do cálculo:

$$
C D=\left[G H+\left(N D P *\left(\frac{\text { renda }}{30}\right)\right)\right] * P S
$$

Onde:

$\mathrm{CD}=$ Custo da doença

$\mathrm{GH}=$ Gastos Hospitalares

NDP $=$ Números de Dias Perdidos de trabalho

Renda $=$ renda mensal

PS = Cobertura de Plano de Saúde Privado

\section{Limitações Metodológicas}

É importante notar que a estimativa considerou apenas os custos diretos, isto é, aqueles rela- 
cionados apenas às despesas no âmbito hospitalar. Os custos indiretos incluiriam as outras perdas socioeconômicas oriundas da morte prematura e a supressão das remunerações geradas pela incapacidade proveniente das intercorrências e complicações da doença do indivíduo portador da patologia e dos seus familiares ${ }^{13}$. Isto significa que a disposição a pagar de um indivíduo para evitar a doença é maior que os gastos incorridos quando este é acometido pela doença, uma vez que o indivíduo está disposto a pagar uma quantia positiva para evitar dor, mal estar e outros incômodos não medidos nos custos da doença ${ }^{3}$.

Os dados disponíveis de remuneração média dos trabalhadores de Manaus somente contabilizam as rendas da população economicamente ativa nas faixas etárias maiores de 14 anos de idade. Logo, a obtenção do custo da doença poderia estar sendo superestimada, haja vista a estimativa ter incluído também as internações hospitalares de indivíduos da população na faixa etária de menores de 14 anos de idade. Supostamente, entendemos que essa opção incluiria os dias perdidos de atividade produtiva dos seus familiares que os acompanharam durante a permanência hospitalar.

Neste estudo, apesar de a literatura especializada mencionar ${ }^{14}$, não se dimensionou a dupla carga de doença no âmbito dos gastos com internações pelas enfermidades atribuíveis a fatores ambientais, ou seja, as patologias associadas. Como exemplo têm-se as doenças cardiovasculares, analisada entre outras dez complicações de saúde no estudo que estimou o impacto econômico associado ao diabetes mellitus no Brasil em $2000^{13}$.

\section{Considerações Éticas}

O estudo foi aprovado pelo Comitê de Ética em Pesquisa da Escola Superior de Saúde da Universidade do Estado do Amazonas.

\section{Resultados}

No período analisado, as doenças fortemente atribuíveis a fatores ambientais na cidade de Manaus representaram $21,0 \%$ do número total das internações hospitalares. Em doze anos registrou-se incremento no número de internações de 57,0\% o que equivale a 6.046 novas hospitalizações. As enfermidades que apresentaram maior variação no número de internações no período foram: dengue $2.700 \%$, doença de Parkinson 900\%, do- enças cardiovasculares $212,4 \%$, diarreias $177 \%$, câncer de traqueia, brônquio e pulmão 160,5\% e hepatite B 124,1\%. Contudo, as infecções respiratórias inferiores, as doenças cardiovasculares e as diarreias foram responsáveis por cerca de $3 / 4$ das internações.

Essas três últimas enfermidades foram também responsáveis por $21,5 \%$ da ocupação dos leitos hospitalares na cidade de Manaus no período analisado. No caso das doenças fortemente atribuíveis a fatores ambientais, o impacto das três enfermidades foi de $77,2 \%$ no tempo de permanência total. Porém, foram as internações por doença de Parkinson, tuberculose, câncer de traqueia, brônquio e pulmão, e o consumo de álcool e drogas que registraram as maiores permanências média em dias: 14,2; 13,4; 12,0 e 11,9, respectivamente. Vale salientar que houve um incremento na permanência média em dias de internações desse grupo de doenças e agravos. As maiores variações na permanência média em dias foram por consumo de álcool e drogas $(108,8 \%)$, vítimas de agressões $(67,2 \%)$ e quedas $(51,5 \%)$.

Em relação aos gastos hospitalares, as doenças fortemente atribuíveis a fatores ambientais totalizaram R $\$ 140.807 .872,90$, o que correspondeu a $27,0 \%$ do total dos gastos hospitalares na cidade. Em doze anos registrou-se incremento de $467,4 \%$ nos gastos em Manaus. No acumulado do período, as internações hospitalares por doenças cardiovasculares e por infecções respiratórias das vias aéreas inferiores responderam por $74,4 \%$ do total dos gastos, ou seja, por R \$ 104.728.225,37.

No último ano analisado essas duas patologias estão entre as seis causas de internação mais onerosas para o Sistema Único de Saúde de Manaus, que são: doenças cardiovasculares ( $\mathrm{R}$ \$ $2.043,44)$, câncer de traqueia, brônquio e pulmão $(\mathrm{R} \$ 1.284,73)$; agressões $(\mathrm{R} \$ 1.152,02)$, acidente de trânsito (R $1.018,74)$, queda $(955,76)$ e infecções respiratórias das vias aéreas inferiores $(R$ \$ $819,65)$. No geral, o valor médio das internações por doenças fortemente atribuíveis a fatores ambientais apresentou variação positiva de 261,4\%, crescendo de $\mathrm{R} \$ 337,78$ em 1998 para $\mathrm{R} \$$ 1.220,66 em 2009.

Conforme observado acima, as doenças cardiovasculares, as infecções respiratórias das vias aéreas inferiores e as diarreias, além de representarem cerca de $3 / 4$ do número de internações, são também responsáveis pelo maior número de dias de ausência da atividade produtiva por internação hospitalar. 
Em 1998, 78,0\% dos dias perdidos foram em decorrência de internação hospitalar por infecções respiratórias das vias aéreas inferiores, doenças cardiovasculares, quedas, acidentes de trânsito e diarreias. Essas doenças e agravos custaram para a cidade $\mathrm{R} \$ 4.739 .659,96$ (ou 78,1\% do total do custo das doenças fortemente atribuíveis a fatores ambientais na cidade). Em 2009, houve uma inversão do primeiro e segundo lugares, ficando em primeiríssimo lugar, as doenças cardiovasculares responsáveis por mais da metade dos dias perdidos $(53,9 \%)$ e dos custos $(62,6 \%)$.

No geral, o custo das doenças fortemente atribuíveis a fatores ambientais na cidade de Manaus, considerados os valores corrigidos pelo Índice Geral de Preços do Mercado para o ano de 2009, foi estimado em $\mathrm{R} \$ 286.852 .666,97$, dos quais as doenças cardiovasculares, as infecções respiratórias das vias aéreas inferiores e as doenças diarreicas foram responsáveis por 78,6\%.

\section{Discussão}

O aumento dos gastos da assistência hospitalar, geralmente, é explicado por uma maior oferta dos serviços de saúde, do progresso tecnológico e do envelhecimento da população ${ }^{15}$. No caso de Manaus, as razões que explicariam o crescimento do número de internações e dos gastos com assistência hospitalar por doenças fortemente atribuíveis a fatores ambientais transcendem essas generalizações.

Por exemplo, no período analisado não se registrou aumento significante do número de estabelecimentos de saúde e de leitos hospitalares. Dados disponíveis no site do Cadastro Nacional de Estabelecimentos de Saúde do Departamento de Informática do Ministério da Saúde (CNES/Datasus) mostram que a oferta de leitos em Manaus, entre os anos de 2006 e 2009, apresentou variação positiva de apenas 1,6\% (o número de leitos em dezembro de 2010 era de 3.708 unidades $)^{16}$. Caso aplicássemos a legislação vigente (Portaria GM/MS 1.101, de 12/06/2002) que preconiza de 2,5 a 3 leitos para cada 1.000 habitantes, Manaus, em 2010, necessitaria de 4.505 a 5.406 leitos $^{16}$. A rede de saúde, em especial da assistência à saúde primária, evocadas pelas estratégias dos programas de agentes comunitários de saúde e saúde da família (PACS/PSF), registrou redução na cobertura de atendimento da população. Estudo recente de Oliveira et al. ${ }^{17}$ constatou um decréscimo na cobertura do PACS/PSF em Manaus de $40,1 \%$ para $34,1 \%$, entre os anos de 2004 e 2008 (no ano de 2009, a redução foi ainda maior: 33,7\%). No período, apesar do estudo ter constatado redução das internações de algumas enfermidades sensíveis às intervenções da atenção primária, os autores não confirmam que tenha sido essa cobertura a responsável. Advertem que o crescimento do número de equipes não implica necessariamente na redução nas internações hospitalares, pois não constataram alterações reais nas tradicionais e pouco efetivas formas de atenção à saúde promovida por esses serviços na cidade.

Já em relação ao crescimento dos gastos com assistência hospitalar, sabe-se, por exemplo, que as despesas com serviços de saúde tendem a crescer sempre mais do que o Produto Interno Bruto do país. A explicação está no fato de que pelo menos quatro fatores agem sobre a demanda: (i) os meios de prevenção e de tratamento das doenças foram se tornando mais sofisticados e onerosos (progresso tecnológico; difusão da inovação); (ii) a ampliação da especialização da área de saúde, em especial com a entrada de novos profissionais egressos dos novos centros formadores da cidade, produziu o fracionamento do cuidado, ampliando o tempo de permanência e ou a reincidência da internação; (iii) fatores culturais, reproduzidos em algumas situações em que o indivíduo ou os familiares induzem o médico a prescrever a internação ${ }^{15,18,19}$; e, por último, (iv) não poderíamos deixar de mencionar, a ausência da assistência à saúde primária capaz de captar precocemente ou rastrear as populações mais vulneráveis aos determinantes socioculturais e biológicos desse grupo de enfermidades aqui estudado.

Cabe destacar, que esse último fator, baseado na constatação acima, constitui uma das principais hipóteses que explicaria o aumento de internações por doenças fortemente atribuíveis a fatores ambientais em doze anos na ordem de 57,0\% em Manaus.

De fato, a cidade de Manaus, a partir dos anos 1970, experimentou uma nova dinâmica econômica impulsionada pela produção industrial da zona franca que reorientou e intensificou as atividades na capital. A concentração de $80 \%$ do PIB e $50 \%$ da população do estado do Amazonas na capital é elucidativa para compreender o inchaço demográfico na cidade. Em 40 anos de vigência da zona franca de Manaus, a população cresceu 5,5 vezes (1970: 311.622 habitantes; 2000: 1.405 .835 habitantes; 2010: 1.718.584 habitantes) $)^{2,20}$.

Segundo o relatório ambiental urbano integrado elaborado pelo Programa das Nações Uni- 
das para o Meio Ambiente (PNUMA), a urbanização sem a necessária ampliação e melhoria da infraestrutura é responsável pela degradação ambiental de Manaus. A cidade apresenta um escasso planejamento territorial urbano, que a torna incapaz de atender às necessidades da população autóctone e muito menos da população migrante, exacerbando problemas relativos à carência de infraestrutura e tensões sociais, com impactos sobre as condições de vida e saúde. Ademais, dentre os problemas de saúde característicos deste contexto, destacam-se, ainda, as doenças infecciosas e parasitárias, combinadas com a poluição, os acidentes e as violências ${ }^{21}$.

A composição da pirâmide etária no comparativo dos dados intercensitários registrou uma sensível redução nas faixas de 0 a 19 anos de idade (em 2000 representava $45 \%$ da população e em 2010 representou $37,9 \%$ do total dos habitantes) e um incremento na faixa superior a 20 anos de idade (em 2000 representava $54,8 \%$ da população; e em 2010 representou $61,9 \%$ do total dos habitantes). Sendo esta última faixa etária mais sensível às doenças cardiovasculares, violências não intencionais (acidentes de trânsito e quedas) e violências intencionais (agressões), a nova composição demográfica se configura como fator de demanda de assistência hospitalar relevante para o contexto da cidade.

No tocante às frações atribuíveis a fatores ambientais, as doenças cardiovasculares respondem por 16\% (IC: 7-23\%), as infecções respiratórias das vias aéreas inferiores por $41 \%$ (IC: 32 $47 \%$ ), e as diarreias por $94 \%$ (IC: $84-98 \%$ ) da carga global das doenças. No caso das doenças cardiovasculares, que responderam em 2009 por mais da metade do custo da doença em Manaus, os principais fatores de risco atribuíveis são: o estilo de vida relacionado ao sedentarismo e aos riscos ocupacionais relacionados a estressores psicossociais e a fadiga física no ambiente de trabalho. Esses fatores de risco são mais atribuíveis às intercorrências hospitalares por doença isquêmica do coração e acidente vascular cerebral na faixa etária de 20-69 anos de idade ${ }^{5}$.

Surpreendentemente, no perfil de morbidade hospitalar, as violências urbanas não se traduzem como fator forte que age sobre a demanda nos serviços hospitalares de Manaus, apesar dos índices de agressões e de acidentes de trânsito fatais figurarem entre os principais coeficientes de anos potenciais de vida perdidos (CAPVP). Dias et al..$^{22}$ identificaram entre os ano de 2000 e 2007, que as agressões fatais foram a principal causa do CAPVP (17,23 e 16,82/1.000 habitantes, respectivamente), enquanto que os acidentes de trânsito no primeiro ano eram a terceira principal causa de morte e no último ano analisado representou a sexta causa, apesar de no período ter-se registrado variação positiva de $20 \%$ destes casos (CAPVP 8,53 e 7,23/1.000 habitantes, respectivamente).

Há três hipóteses que explicariam o fenômeno: (i) a ocorrência do óbito dos vitimados em acidentes de trânsito e quedas no próprio local do evento; (ii) sub-registro dos profissionais de saúde, por temerem retaliação por parte do agressor ou da vítima da agressão; (iii) desconhecimento dos profissionais da Portaria SAS/MS n. ${ }^{\circ}$ 142, de 13 de novembro de 1997 e da Portaria GM/MS n. ${ }^{\circ}$ 1.969, de 25 de outubro de 2001 que tornaram obrigatórias o registro dos códigos do Capitulo XX (Causas Externas de Morbidade e Mortalidade) da CID10 no diagnóstico secundário do campo da $\mathrm{AIH}^{23,24}$.

Diante do exposto, não é possível falar de transição demográfica, nem de transição epidemiológica, concepções formuladas por volta de 1970 para descrever as mudanças nas características de saúde das sociedades em desenvolvimento antes e durante a transição demográfica na Europa. Estamos mais propensos a concordar com Smith e Ezzati ${ }^{25}$ que, ao abordarem os efeitos do desenvolvimento econômico nas mais distintas sociedades, propuseram fazê-lo avaliando os diferentes padrões de riscos. Assim, a situação de Manaus poderia ser vinculada ao processo de desenvolvimento associado à urbanização e industrialização, bem como à expansão das fronteiras agrícolas, cujos produtos e subprodutos tóxicos e poluentes resultam em múltiplas consequências à saúde das comunidades e dos trabalhadores.

É bastante característico nesse padrão de risco, considerar que mesmo efeitos diretos ou mediados sobre a saúde e mensuráveis na atualidade podem resultar de múltiplas interações ao final de uma longa e complexa rede de eventos e situações, combinados em diferentes níveis e escalas espaciais e temporais ${ }^{26}$.

Vale, ainda, destacar, três importantes endemias, com alta incidência na Amazônia Legal, e em Manaus, mas de baixa demanda hospitalar: tuberculose, malária e hanseníase. A primeira é a tuberculose que está intimamente ligada à desigualdade social, ao envelhecimento da população, aos grandes movimentos migratórios, à urbanização acelerada e ao advento da AIDS. A internação somente é indicada em casos de manifestações graves, intolerância medicamentosa e 
associação com outras doenças. No Brasil as internações prolongadas são verificadas em pacientes com comorbidade, como por exemplo, em paciente com AIDS, por se tratar da segunda principal infecção oportunista nestes pacientes ${ }^{27}$.

A segunda é a malária em que os estados do Acre, Amapá, Amazonas, Pará, Rondônia e Roraima registram $97 \%$ do total de casos do país. A internação hospitalar somente é indicada para pacientes portadores da malária causada pelo Plasmodium falciparum ou que apresentem sinais e sintomas graves da doença. Segundo relatório do Tribunal de Contas da União (TCU), entre os anos de 2002 e 2007, o valor médio no Brasil de uma internação hospitalar por malária era de R\$166,00. Nesse período foram economizados cerca de R \$ 6,8 milhões oriundos de quarenta mil internações evitadas, em virtude da intensificação das ações do Programa Nacional de Controle da Malária (PNCM) ${ }^{28}$.

A terceira e última é a hanseníase que, apesar de o estudo denominado Reducing risk, promoting healthy life ${ }^{11}$ não configurá-la como uma FAMA, pesquisas brasileiras recentes em cinco importantes municípios da Amazônia Legal revelaram a predominância de fatores ambientais na sua determinação social e em casos de recidiva. São exemplos desses fatores a "periferização" recente da cidade, a residência em casa alugada, a habitação em casa de madeira/taipa, a coabitação com mais de cinco pessoas no mesmo domicílio e o acesso ao serviço de saúde. A cidade de Manaus, em 2004, foi responsável por $46,2 \%$ dos casos novos e por $42,3 \%$ dos casos em registro ativo de todo o Amazonas, sendo a doença classificada como hiperendêmica ${ }^{29,30}$. Atualmente as ações de controle da hanseníase são ambulatoriais e a internação hospitalar ocorre somente decorrente de intercorrências da doença (reação à quimioterapia, cirurgia) ou por indicação social, oriunda de abandono ou preconceito dos familiares, em instituições especializadas, o que se aplica também à tuberculose ${ }^{31}$.

Diante do exposto, podemos indagar: de que depende a decisão política, sob uma perspectiva da economia e do bem estar da população, para reverter a lógica da aplicação dos escassos recursos financeiros públicos de modo a favorecer o controle do acometimento de enfermidades sensíveis a fatores ambientais?

\section{Colaboradores}

MS Medeiros, DS Sacramento, JC Hurtado-Guerrero, RA Ortiz e ALD Fenner participaram do delineamento da pesquisa, interpretação e discussão dos dados da versão submetida para avaliação da revista. MS Medeiros participou da concepção, programação do tabulador e realizou as descrições dos resultados e discussões. DS Sacramento programou, coletou, tabulou e cálculo os dados em planilhas eletrônicas. JC HurtadoGuerrero realizou o cálculo dos dados em planilhas eletrônicas e revisou a descrição da discussão. RA Ortiz é autor da metodologia, calibrou os dados e revisou as descrições dos resultados. ALD Fenner participou das descrições da introdução e dos resultados do estudo.

\section{Agradecimentos}

A equipe responsável pelo sistema de Relação Anual de Informações Sociais (RAIS) do Ministério do Trabalho e Emprego (MTE) e ao Sr. Jacques Levin do Fórum de discussão do Departamento de Informática do Sistema Único de Saúde do Ministério da Saúde. 


\section{Referências}

1. Celentano D, Santos D, Veríssimo A. A Amazônia e os objetivos do Milênio. Belém: Imazon; 2010.

2. Freitas CM, Giatti LL. Indicadores de sustentabilidade ambiental e de saúde na Amazônia Legal, Brasil. Cad Saude Publica 2009; 25(6):1251-1266.

3. Motta RS, Ortiz RA, Ferreira SF. Avaliação econômica dos impactos causados pela poluição atmosférica na saúde humana: Um estudo de caso para São Paulo. [página na internet]. 1998 [acessado $2010 \mathrm{dez} 22$ ]; [cerca de 19 p.]. Disponível em: http://www.race. nuca.ie.ufrj.br/eco/trabalhos/mesa3/5.doc.

4. Vicentin G, Minayo CG. Saúde, ambiente e desenvolvimento econômico na Amazônia. Cien Saude Colet 2003; 8(4):1069-1085.

5. Prüss-Üstün A, Corvalán C. Preventing disease through healthy environments. Towards an estimate of the environmental burden of disease. Geneva: World Health Organization (WHO); 2006.

6. Cortez-Pinto H, Gouveia M, Pinheiro LS, Costa J, Borges M, Carneiro AV. The burden of disease and the cost of illness attributable to alcohol drinking results of a national study. Alcohol Clin Exp Res 2010; 34(8):1442-1449.

7. Magalhães M. O narcotráfico. São Paulo: Publifolha; 2000

8. Carvalho AXY, Cerqueira D. Estimação da perda de produção devido a mortes por causas externas nas cidades brasileiras. In: Carvalho AXY, Albuquerque CW, Mota JA, Piancastelli M, organizadores. Ensaios de economia regional e urbana. Brasília: IPEA; 2007. p. 64-105.

9. Smith KR, Corvalán CF, Kjellström T. How Much Global Ill Health Is Attributable to Environmental Factors? Epidemiology 1999; 10(5):573-584.

10. Corrêa CRP, Barreto SM, Passos VMA. Métodos de estimativa da mortalidade atribuível ao tabagismo: uma revisão da literatura. Epidemiol. Serv. Saúde 2008; 17(10):43-57.

11. World Health Organization (WHO). World Health Report 2002 - Reducing risks, promoting healthy life. Geneva: WHO; 2002.

12. Murray C, Lopez AD, Mathers CD, Stein C. The Global Burden of Disease 2000 project: aims, metho$\mathrm{ds}$ and data sources [página na internet]. [acessado 2009 mar 15]; [cerca de 57 p.]. Disponível em: www.who.int/entity/healthinfo/paper36.pdf.

13. Vianna CMM, Souza AM, Vianna D, Mosegui GBG, Pereira MHS. Estimativa do impacto econômico associado ao diabetes mellitus no Brasil para o ano de 2000 [página na internet]. 2002 [acessado 2011 set 14]; [cerca de 19 p]. Disponível em: www.ppge.ufrgs.br/ ats/disciplinas/2/vianna-souza-vianna-2002.pdf

14. Schramm JMA, Oliveira AF, Leite IC, Valente JG, Gadelha AMJ, Portela MC, Campos, MR. Transição epidemiológica e o estudo de carga de doença no Brasil. Cien Saude Colet 2004; 9(4):897-908.

15. Zucchi P, Del Nero C, Malik AM. Gastos em saúde: os fatores que agem na demanda e na oferta dos serviços de saúde. Saude e Sociedade 2000; 9(1/2):127150.
16. Brasil. Cadastro Nacional de Estabelecimentos de Saúde. Hospitalar - Leitos Internação, 2011. [página na internet]. [acessado 2011 out 05]. Disponível em: http://www2.datasus.gov.br/DATASUS/index.php? area $=0204 \mathrm{eid}=11665 \mathrm{eVObj}=\mathrm{http}: / /$ tabnet.datasus gov.br/cgi/deftohtm.exe?cnes/cnv/leiint.

17. Oliveira HM, Gonçalves MJF, Pires ROM. Caracterização da Estratégia Saúde da Família no Estado do Amazonas, Brasil: análise da implantação e impacto. Cad Saude Publica 2011; 27(1):35-45.

18. Vecine Neto G, Malik AM. Tendências na assistência hospitalar. Cien Saude Colet 2007; 12(4):825-839.

19. Serra J. A questão da saúde no Brasil: Entrevista com José Serra. Estud. av. 1999; 13(35):39-50.

20. Brasil. Instituto Brasileiro de Geografia e Estatística. Censo Demográfico. 2000 e 2010. [página na internet]. 2011 [acessado 2011 maio 30]. Disponível em: http://www.censo2010.ibge.gov.br/sinopse/ webservice/frm_piramide.php?ano $=2010$ ecodigo $=130260$ ecorhomem $=88 \mathrm{C} 2 \mathrm{E} 6$ ecormulher $=$ F9C 189 ewmaxbarra $=180$.

21. Velloso R. Projeto GEO cidades: relatório ambiental urbano integrado: informe GEO Manaus. Rio de Janeiro: Consórcio Parceria 21; 2002.

22. Dias PBD, Medeiros MS, Hurtado-Guerrero JC. Anos potenciais de vida perdidos atribuíveis a fatores ambientais na cidade de Manaus, Amazonas, Brasil. In: Anais da VII Jornada de IC do CPqLMD; 2010; Manaus. p. 17-22.

23. Oliveira LR, Mello Jorge MHP. Análise epidemiológica das causas externas em unidades de urgência e emergência em Cuiabá/Mato Grosso. Rev Bras Epidemiol 2008; 11(3):420-430.

24. Tomimatsu MFAI, Andrade SM, Soares DA, Mathias TAF, Sapata MPM, Soares DFPP, Souza RKT. Qualidade de causa externa, dados do sistema de informações hospitalares. Rev Saude Publica 2009; 43(3):413-420.

25. Smith KR, Ezzati M. How environmental health risks change with development: The Epidemiologic and Environmental Risk Transitions Revisited. Annu Rev Environ Resour 2005; 30:291-333.

26. Hales S, Butler C, Alistair W, Corvalan C. Health aspects of the Millennium Ecosystem Assessment. Ecohealth 2004; 1(2):124-128.

27. Assunção CG, Seabra JDR, Figueiredo RM. Percepção do paciente com tuberculose sobre a internação em hospital especializado. Ciencia y enfermeria 2009; 15(2):69-77.

28. Brasil. Ministério da Saúde (MS). Resultados e conquistas. Mais Saúde. Direito de todos. Uma prestação de contas à sociedade. [página na internet]. 2009 [acessado 2011 out 28]. Disponível em: http:/ /bvsms.saude.gov.br/bvs/pacsaude/pdf/resultados_ conquistas_mais_saude.pdf.

29. Ferreira SMB, Ignotti E, Gamba MA. Fatores associados à recidiva em hanseníase em Mato Grosso. Rev Saude Publica 2011; 45(4):756-764. 
30. Imbiriba ENB, Silva Neto AL, Souza WV, Pedroza V, Cunha MG, Garnelo L. Desigualdade social, crescimento urbano e hanseníase em Manaus: abordagem espacial. Rev Saude Publica 2009; 43(4):656-665.

31. Soares CGM. Hanseníase no estado do Pará: perfil epidemiológico da população que demanda internação por reações hansênicas [dissertação]. Belém: Escola Nacional de Saúde Pública; 2001.

Artigo apresentado em 30/11/2012

Aprovado em 02/02/2013

Versão final apresentada em 15/02/2013 